\title{
A SUPER-SOLAR METALLICITY FOR THE PROGENITOR OF KEPLER'S SUPERNOVA
}

\author{
Sangwook Park ${ }^{1}$, Carles Badenes ${ }^{2}$, Koti Mori $^{3}$, Ryohei Kaida ${ }^{3}$, Eduardo Bravo ${ }^{4}$, Andrew Schenck ${ }^{1}$, \\ Kristoffer A. Eriksen ${ }^{5}$, John P. Hughes ${ }^{6}$, Patrick O. Slane ${ }^{7}$, David N. Burrows ${ }^{8}$, and Jae-Joon Lee ${ }^{9}$ \\ ${ }^{1}$ Department of Physics, University of Texas at Arlington, Box 19059, Arlington, TX 76019, USA; s.park @ uta.edu \\ ${ }^{2}$ Department of Physics and Astronomy and Pittsburgh Particle Physics, Astrophysics, and Cosmology Center (PITT-PACC), University of Pittsburgh, \\ 3941 O’Hara Street, Pittsburgh, PA 15260, USA; badenes@ pitt.edu \\ ${ }^{3}$ Department of Applied Physics, University of Miyazaki, 1-1 Gakuen Kibana-dai Nishi, Miyazaki 889-2192, Japan \\ ${ }^{4}$ Department Física i Enginyeria Nuclear, Univ. Politécnica de Catalunya, Carrer Pere Serra 1-15, E-08173 Sant Cugat del Vallés, Spain \\ 5 XDT-6, Los Alamos National Laboratory, P.O. Box 1663, Los Alamos, NM 87545, USA \\ ${ }^{6}$ Department of Physics and Astronomy, Rutgers University, 136 Frelinghuysen Road, Piscataway, NJ 08854-8019, USA \\ ${ }^{7}$ Harvard-Smithsonian Center for Astrophysics, 60 Garden Street, Cambridge, MA 02138, USA \\ ${ }^{8}$ Department of Astronomy and Astrophysics, Pennsylvania State University, 525 Davey Laboratory, University Park, PA 16802, USA \\ ${ }^{9}$ Korea Astronomy and Space Science Institute, Daejeon 305-348, Republic of Korea \\ Received 2013 February 20; accepted 2013 February 27; published 2013 March 26
}

\begin{abstract}
We have performed deep X-ray observations of the remnant of Kepler's supernova (SN 1604) as a Key Project of the Suzaku Observatory. Our main goal is to detect secondary Fe-peak elements in the supernova (SN) ejecta to gain insights into the Type Ia SN explosion mechanism and the nature of the progenitor. Here, we report our initial results. We made a conclusive detection of X-ray emission lines from highly ionized $\mathrm{Mn}, \mathrm{Cr}$, and $\mathrm{Ni}$ as well as Fe. The observed Mn-to-Cr line flux ratio is $\sim 0.60, \sim 30 \%$ larger than that measured in Tycho's remnant. We estimate an Mn-to-Cr mass ratio of $\sim 0.77$, which is strongly suggestive of a large neutron excess in the progenitor star before the onset of the thermonuclear runaway. The observed Ni-to-Fe line flux ratio $(\sim 0.03)$ corresponds to a mass ratio of $\sim 0.06$, which is generally consistent with the products of the explosive Si-burning regime in Type Ia explosion models, and rules out contamination from the products of neutron-rich nuclear statistical equilibrium in the shocked ejecta. Together with the previously suggested luminous nature of the explosion, these mass ratios provide strong evidence for a super-solar metallicity in the SN progenitor $\left(\sim 3 Z_{\odot}\right)$. Kepler's SN was likely the thermonuclear explosion of a white dwarf formed in the recent past that must have exploded through a relatively prompt channel.
\end{abstract}

Key words: ISM: individual objects (Kepler's supernova remnants) - ISM: supernova remnants - X-rays: ISM

\section{INTRODUCTION}

The progenitors of Type Ia supernovae ( $\mathrm{SNe}$ ) are believed to be $\mathrm{C} / \mathrm{O}$ white dwarfs (WDs) in close binary systems. The WD becomes unstable when it approaches the Chandrasekhar limit $\left(M_{\mathrm{Ch}}=1.44 M_{\odot}\right)$ by either accreting a sufficient amount of material from a non-degenerate companion (single-degenerate channel) or merging with another WD (double-degenerate channel). This mass increase triggers a thermonuclear runaway and an explosion ensues by the fusion of carbon and oxygen. While this is generally accepted, the nature of the progenitor and its binary companion and the details of the explosion process remain elusive. The progenitor's nature (e.g., age, mass, and metallicity), its environment (e.g., ambient structure and the companion's nature), and the details of the explosion mechanism (whether the burning front is a deflagration, a delayed detonations, etc.) may significantly vary among $\mathrm{SNe}$ Ia. Understanding the diversity of SN Ia is essential to reduce systematic uncertainties in their cosmological applications. It has been established that SN Ia progenitors have a wide range of delay times, from a few hundred Myr to several Gyr, and that "prompt" SNe Ia produce more ${ }^{56} \mathrm{Ni}$ and are brighter than the "delayed" population (e.g., Maoz \& Mannucci 2012). If SN Ia exploded with delay times of only a few hundred Myr, their progenitor's masses must have been significantly larger $\left(\sim 3.5-8 M_{\odot}\right)$ than the Sun in their main-sequence stage (Aubourg et al. 2008).

The progenitor's metallicity $(Z)$ is a key parameter related to the age population of SNe Ia. During the main-sequence evolution of an intermediate-mass star, the $\mathrm{CNO}$ cycle converts $\mathrm{C}, \mathrm{N}$, and $\mathrm{O}$ into ${ }^{14} \mathrm{~N}$, which subsequently produces ${ }^{22} \mathrm{Ne}$ through the intermediate $\beta^{+}$decay of ${ }^{18} \mathrm{~F}$ in the star's hydrostatic He-fusion phase. This results in neutron excess $\left(\eta=1-2 Y_{e}\right.$, where $Y_{e}$ is the proton to nucleon number ratio) in the progenitor. Except for the deepest core of $M<0.2 M_{\odot}$, where $\eta$ is dominated by electron capture in neutron-rich nuclear statistical equilibrium ( $n$-rich NSE; Brachwitz et al. 2000), $\eta$ is proportional to $Z$ (Timmes et al. 2003). During the explosion, the excess amount of neutrons is stored in trace elements with an unequal number of protons and neutrons. ${ }^{55} \mathrm{Mn}$ is the most abundant among such elements. ${ }^{52} \mathrm{Cr}$ is produced by the same explosive Si-burning as Mn, but its synthesis is unrelated to $\eta$. Thus, the Mn-to-Cr mass ratio is an excellent tracer of the progenitor's $Z$ (Badenes et al. 2008; Bravo 2013). The progenitor's $Z$, together with the details of the explosion physics, can also influence the synthesis of stable $\mathrm{Ni}$ (mostly ${ }^{58} \mathrm{Ni}$; e.g., Iwamoto et al. 1999; Timmes et al. 2003). Observational constraints on the content of these Fe-group ejecta elements in SNe Ia would thus provide insights on the detailed nature of the progenitor system and the explosion mechanism. However, the relatively long decay times of radioactive Fe-peak elements ( $\sim$ yr; e.g., Kuchner et al. 1994) make it difficult to detect these elements directly in $\mathrm{SNe}$ Ia. Instead young supernova remnants (SNRs) provide a useful opportunity.

Kepler's SNR ("Kepler" hereafter) is the remnant of SN 1604. The historical SN light curve suggested a Type Ia explosion (Baade 1943), although this was not a unique classification (Doggett \& Branch 1985). Kepler shows strong atomic X-ray emission lines from highly ionized ions of $\mathrm{Si}, \mathrm{S}, \mathrm{Ca}$, and $\mathrm{Fe}$ 
(e.g., Decourchelle \& Ballet 1994; Kinugasa \& Tsunemi 1999; Cassam-Chenaï et al. 2004). These overall X-ray spectral characteristics are similar to those of Tycho's SNR, an unambiguous Type Ia (Krause et al. 2008; Badenes et al. 2006). Reynolds et al. (2007) found that the X-ray ejecta are dominated by Si, S, and $\mathrm{Fe}$, without evidence for $\mathrm{O}, \mathrm{Ne}$, and $\mathrm{Mg}$. Based on this ejecta abundance structure and the absence of a conspicuous stellar remnant (see also Blair 2005), they ruled out a core-collapse origin for Kepler. Kepler appears to be interacting with a modified circumstellar medium (CSM; Bandiera 1987). Overabundant nitrogen was detected in Kepler, indicating the presence of dense CSM produced by massive stellar winds (Dennefeld 1982; Blair et al. 1991). The spatial distribution of interacting CSM suggested that the strong winds might have originated from an asymptotic giant branch (AGB) companion (Chiotellis et al. 2012; Burkey et al. 2013). These results generally support a single-degenerate origin for Kepler.

Based on observations at several wavebands, a range of distances (from $d \sim 3 \mathrm{kpc}$ to $d>6 \mathrm{kpc}$ ) to Kepler has been suggested (e.g., Reynoso \& Goss 1999; Sankrit et al. 2005; Aharonian et al. 2008; Vink 2008). Recently, Patnaude et al. (2012) showed that hydrodynamic and X-ray spectral simulations are consistent with the Chandra spectrum of Kepler for $d \gtrsim 7 \mathrm{kpc}$ if the shock is expanding into massive stellar winds, while the distance may be closer $(d \sim 4 \mathrm{kpc})$ for the case of a uniform medium. The Type Ia explosion must have been bright and ${ }^{56} \mathrm{Ni}$-rich to explain the strong Fe line emission observed by Chandra (Patnaude et al. 2012).

With its Type Ia origin, young age (409 yr), and the ejecta-dominated spectrum of X-ray emission, Kepler provides an excellent opportunity to study the details of Type Ia explosion mechanisms and the progenitor's nature. In this Letter, we report the initial results from our Suzaku Key Project of Kepler. As we presented in our preliminary report (Park et al. 2012), we detect $\mathrm{X}$-ray emission from highly ionized $\mathrm{Mn}, \mathrm{Cr}, \mathrm{Ni}$, and $\mathrm{Fe}$ ions. Line flux ratios of these $\mathrm{Fe}$-group elements strongly suggest a super-solar $Z$ for the progenitor. We describe our observations in Section 2. Our data analysis and results are presented in Section 3. A discussion and conclusions are presented in Section 4.

\section{OBSERVATIONS}

We performed our Suzaku observations of Kepler as part of a Key Project in 2009 August-2011 March during Cycles 4-5 (Table 1). ${ }^{10}$ The X-ray imaging spectrometer (XIS) was chosen to be the primary detector. The FI-CCDs (XIS0 and 3) have a better energy resolution (FWHM $\sim 180 \mathrm{eV}$ at $E \sim 6 \mathrm{keV}$ ) than the BI-CCD (XIS1, FWHM $\sim 250 \mathrm{eV}$ at $E \sim 6 \mathrm{keV})$. The non-X-ray background flux is significantly lower (by a factor of $\sim 2-3$ at $E=5-8 \mathrm{keV}$ ) in the FI-CCDs than the BI-CCD. When the two available FI-CCDs (XISO and 3 ) are combined, the collecting area is larger by a factor of two than that of XIS1. Thanks to the good energy resolution and low background, the FI-CCDs efficiently detect and measure faint line emission fluxes from Fe-group ejecta elements at $E \sim 5-8 \mathrm{keV}$, which is our primary goal. Thus, we used the XIS0 and 3 data in this work. Some time intervals in observations taken in 2011 March were excluded from the analysis because of temporary on-board software issues with the XISO (for $\sim 25 \mathrm{ks}$ in ObsID 505092050). Otherwise, we followed the standard data

\footnotetext{
${ }^{10}$ We also used our earlier Suzaku data (ObsID 502078010), performed on
} 2008 February.
Table 1

Suzaku Observation Log of Kepler's Supernova Remnant

\begin{tabular}{lcccr}
\hline \hline ObsID & Date & $\begin{array}{l}\text { Target } \\
\text { Name }\end{array}$ & $\begin{array}{c}\text { Pointing } \\
\left(\alpha_{2000}\left({ }^{\circ}\right), \delta_{2000}\left({ }^{\circ}\right)\right)\end{array}$ & $\begin{array}{c}\text { Exposure } \\
(\mathrm{ks})\end{array}$ \\
\hline 502078010 & 2008 Feb 18 & Kepler & $262.6757,-21.4668$ & 117.0 \\
504101010 & 2009 Sep 13 & Kepler_BG_GE & $263.5352,-20.2853$ & 47.0 \\
504101020 & 2009 Aug 29 & Kepler_BG_GE & $263.5352,-20.2853$ & 24.7 \\
504103010 & 2009 Oct 1 & Kepler_BG_GN & $261.9150,-20.2528$ & 68.6 \\
504104010 & 2009 Oct 3 & Kepler_BG_GS & $263.1005,-22.9710$ & 67.3 \\
504102010 & 2009 Oct 5 & Kepler_BG_GW & $261.8058,-22.7903$ & 65.3 \\
505092010 & 2010 Sep 30 & Kepler SNR & $262.6746,-21.5263$ & 17.7 \\
505092020 & 2010 Oct 6 & Kepler SNR & $262.6739,-21.5293$ & 111.2 \\
505092030 & 2011 Feb 23 & Kepler SNR & $262.6746,-21.4606$ & 34.2 \\
505092040 & 2011 Feb 28 & Kepler SNR & $262.6730,-21.4605$ & 146.2 \\
505092050 & 2011 Mar 8 & Kepler SNR & $262.6732,-21.4607$ & 84.6 \\
505092060 & 2011 Mar 14 & Kepler SNR & $262.6734,-21.4607$ & 46.5 \\
505092070 & 2011 Mar 29 & Kepler SNR & $262.6738,-21.4631$ & 133.4 \\
\hline
\end{tabular}

reduction process including charge injection, and combined all $3 \times 3$ and $5 \times 5$ mode spectra for each XIS0 and XIS3. After the data reduction, the total effective exposures are $\sim 665$ and $\sim 691 \mathrm{ks}$ for XIS0 and XIS3, respectively.

Since Kepler is a bright extended source ( $\sim 2^{\prime}$ in radius), scattered photons from the SNR dominate nearly the entire CCD due to the large point-spread function of the telescope, which makes it difficult to adequately estimate the background spectrum from surrounding regions. The characterization of the background spectrum is critical to accurately measure fluxes from faint line features. To mitigate the bright scattered light from the SNR, we have performed separate background observations. We chose four nearby source-free regions ( $\sim 1.5$ from Kepler), one each of which is to north, south, east, and west of Kepler (Table 1). We processed these background observations in the same way as we did for the source observations. After the data reduction, the total effective exposure for the background observations is $\sim 273$ ks each for XIS0 and XIS3. We note that recently Yang et al. (2013) analyzed our Suzaku data of Kepler using background estimates from source-free regions on the same XIS chip instead of using separate background pointings. Their measurements of lower fluxes (by $\sim 40 \%$ ) for $\mathrm{Mn} \mathrm{K} \alpha$ and $\mathrm{Cr} \mathrm{K} \alpha$ lines than ours (see Section 3) are likely caused by the scattered X-ray contamination in their background estimates. They suggested a carbon deflagration for Kepler's explosion based on a low value of the Cr-to-Fe $\mathrm{K} \alpha$ line equivalent width (EW) ratio ( 0.008). Our larger $\mathrm{Cr}$ line flux indicates the $\mathrm{Cr}$-to-Fe $\mathrm{K} \alpha$ line $\mathrm{EW}$ ratio of $\sim 0.016$ which would rather favor delayed detonations for Kepler as are self-consistently suggested by our Ni-to-Fe mass ratio measurement (Section 4).

\section{ANALYSIS AND RESULTS}

We extracted the source spectrum from the entire SNR (a circular region of $4^{\prime}$ in radius). We extracted the background spectrum from the same detector region as the source in each background pointing, and combined them. In the 5-8 keV band, we obtained $\sim 130,000$ counts from the source for each of XIS0 and XIS3. The background contribution is $\sim 7 \%$. For the spectral model fits, we re-binned the source spectrum to contain a minimum of 50 counts per energy channel. Our main goal is to measure line fluxes from $\mathrm{Cr}, \mathrm{Mn}, \mathrm{Fe}$, and $\mathrm{Ni}$ using the integrated X-ray spectrum of Kepler. Thus, we fit the backgroundsubtracted spectrum with a phenomenological model in the 5-8 keV band. We fit the observed spectrum with an absorbed 


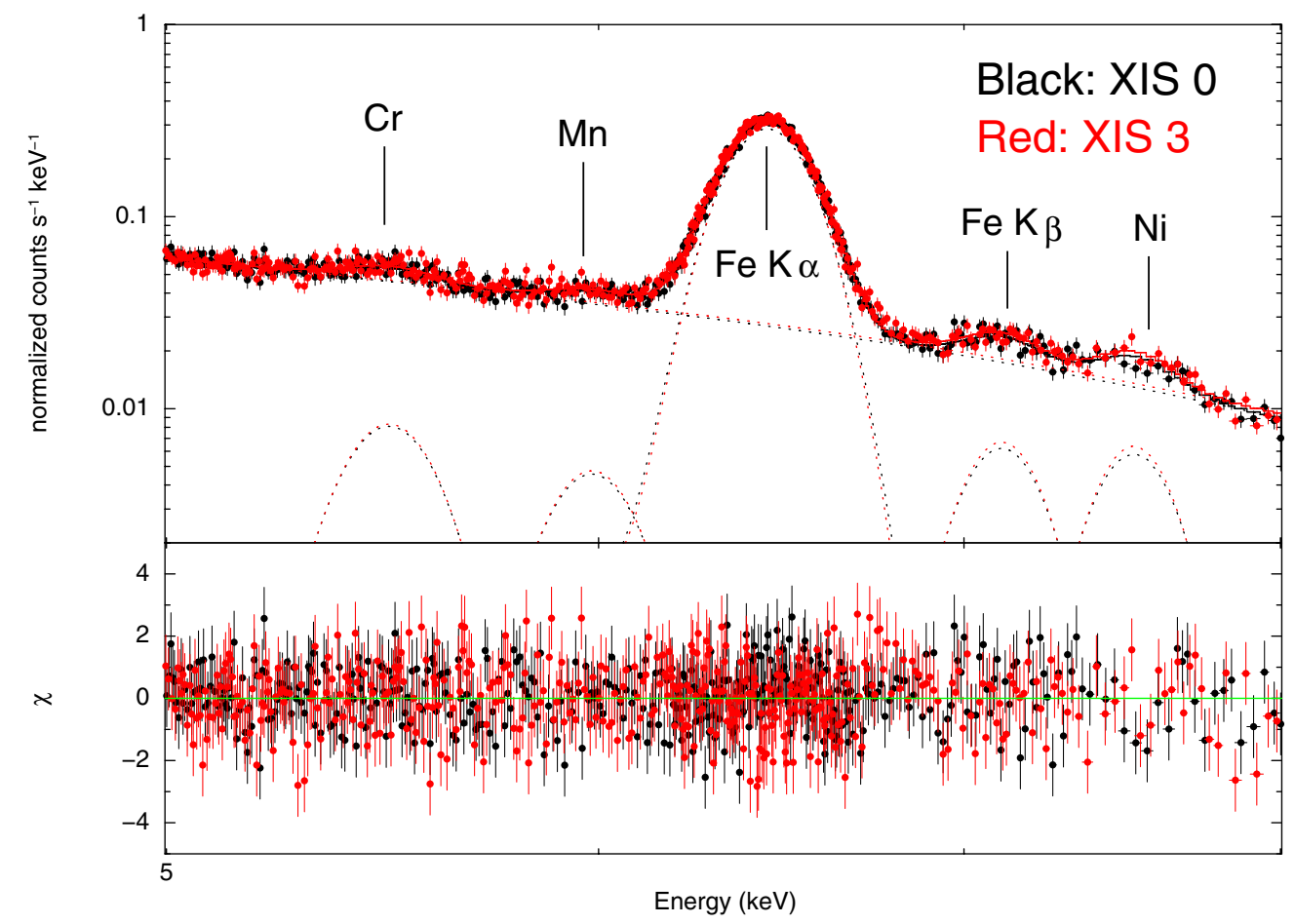

Figure 1. Suzaku XIS spectrum of Kepler's SNR. The best-fit model (PL + 5 Gaussians) is overlaid.

Table 2

Summary of Detected Atomic Emission Lines

\begin{tabular}{lccl}
\hline $\begin{array}{l}\text { Line Center Energy } \\
(\mathrm{keV})\end{array}$ & $\begin{array}{c}\text { Line Width } \\
(\mathrm{eV})\end{array}$ & $\begin{array}{c}\text { Line Flux } \\
\left(10^{-6} \text { photon } \mathrm{cm}^{-2} \mathrm{~s}^{-1}\right)\end{array}$ & Element \\
\hline $5.50 \pm 0.02$ & $65.5 \pm 1.2$ & $8.31 \pm 1.20$ & $\mathrm{Cr}(\mathrm{K} \alpha$ line $)$ \\
$5.99 \pm 0.04$ & $65.5 \pm 1.2$ & $4.99 \pm 1.16$ & $\mathrm{Mn}(\mathrm{K} \alpha$ line $)$ \\
$6.449 \pm 0.0008$ & $65.5 \pm 1.2$ & $365.91 \pm 2.78$ & $\mathrm{Fe}(\mathrm{K} \alpha$ line $)$ \\
$7.12 \pm 0.02$ & $65.5 \pm 1.2$ & $9.92 \pm 1.24$ & $\mathrm{Fe}(\mathrm{K} \beta$ line $)$ \\
$7.53 \pm 0.02$ & $65.5 \pm 1.2$ & $11.18 \pm 1.88$ & $\mathrm{Ni}(\mathrm{K} \alpha$ line $)$ \\
\hline
\end{tabular}

Notes. $2 \sigma$ uncertainties are shown.

a The line width is tied among all lines.

power-law (PL) model with five Gaussian components. The PL component is to characterize the underlying continuum. Each Gaussian is to fit the emission feature from $\mathrm{Cr} \mathrm{K} \alpha(E \sim 5.5 \mathrm{keV})$, $\mathrm{Mn} \mathrm{K} \alpha(E \sim 5.9 \mathrm{keV}), \mathrm{Fe} \mathrm{K} \alpha(E \sim 6.5 \mathrm{keV}), \mathrm{Fe} \mathrm{K} \beta(E \sim$ $7.1 \mathrm{keV})$, and $\mathrm{Ni} \mathrm{K} \alpha(E \sim 7.5 \mathrm{keV})$ lines, respectively. The same Gaussian line width was assumed for all five lines, and was varied to give the best fit. The foreground column is fixed at $N_{\mathrm{H}}=5.2 \times 10^{21} \mathrm{~cm}^{-2}$ (Reynolds et al. 2007). The photon index $(\Gamma)$ and normalization parameters for the PL and Gaussian components are varied freely. We fit the XIS0 and XIS3 spectra simultaneously, tying all parameters between the two spectra.

The observed spectrum and the best-fit model $(\Gamma=2.60$, $\left.\chi^{2} / v=1469.8 / 1513\right)$ are shown in Figure 1 . In the $5-8 \mathrm{keV}$ band, X-ray emission is dominated by the strong $\mathrm{Fe} \mathrm{K} \alpha$ line at $E \sim 6.45 \mathrm{keV}$. X-ray emission lines from He-like ions of $\mathrm{Mn}$ and $\mathrm{Cr}$ are clearly detected (at $\sim 9 \sigma$ and $\sim 14 \sigma$ level for $\mathrm{Mn}$ and $\mathrm{Cr}$, respectively, Table 2 and Figure 1$)$. Fe $\mathrm{K} \beta(E \sim$ $7.1 \mathrm{keV})$ and $\mathrm{Ni} \mathrm{K} \alpha(E \sim 7.5 \mathrm{keV})$ lines are also conclusively detected. To verify that the Ni line was not due to the instrumental Ni fluorescent line, which varies with the incidental cosmic-ray flux, we repeated our Ni line flux measurement using an off-source background region from the on-target data. We found that the Ni line was clearly present with this background subtraction. We also compared the instrumental $\mathrm{Au}$ Ly $\alpha$ line flux $(E=9.671 \mathrm{keV})$ between the source and background observations. These tests indicated only a $\sim 10 \%-30 \%$ difference in the instrumental line fluxes between the source and background pointings. Thus, we conclude that the detected $\mathrm{Ni}$ line originates from Kepler. The small difference in the instrumental background does not affect our discussion in Section 4. Alternatively, we fit the underlying continuum with a thermal bremsstrahlung model $(k T=5.11 \mathrm{keV}$, $\left.\chi^{2} / v=1465.2 / 1513\right)$. The estimated fluxes for Fe and Ni lines are identical to those from the PL model fit. Line fluxes from $\mathrm{Mn}$ and $\mathrm{Cr}$ are estimated to be slightly smaller (by $\sim 10 \%$ ), but the flux ratio is consistent (within $1 \sigma$ uncertainties) with that estimated by the PL model fit.

The archival Chandra data of Kepler show thin non-thermal filaments along the outermost boundary, which are identified as synchrotron radiation from the shock-accelerated relativistic electrons (Reynolds et al. 2007). To test the effect from synchrotron emission in the measured line fluxes, we repeated our spectral model fit with a PL + thermal bremsstrahlung + 5 Gaussians. Based on PL model fits of several non-thermal filaments using the archival Chandra data of Kepler, we estimated $\Gamma \sim$ 2.4-2.6 for these features. Thus, we performed the model fits with $\Gamma$ fixed at 2.4, 2.5, and 2.6. Using the archival Chandra data of Kepler, we estimated that the thin non-thermal shell (typically with a width of $\sim 5^{\prime \prime}$ ) all around the SNR contributes $\sim 10 \%$ of photon counts in the $5-8 \mathrm{keV}$ band. Thus, we assumed a $10 \%$ fractional contribution from the PL component in the 5-8 keV band total flux. These model fits are statistically good $\left(\chi^{2} / v \sim 1.0\right)$ with negligible changes in the best-fit parameters from those described above. The best-fit thermal bremsstrahlung temperature is $k T=5.1 \mathrm{keV}$, and the estimated line fluxes are consistent with the results derived above. We conclude that our measurements of individual line fluxes are robust, and hereafter our discussion is based on the results listed in Table 2 . 
(a)

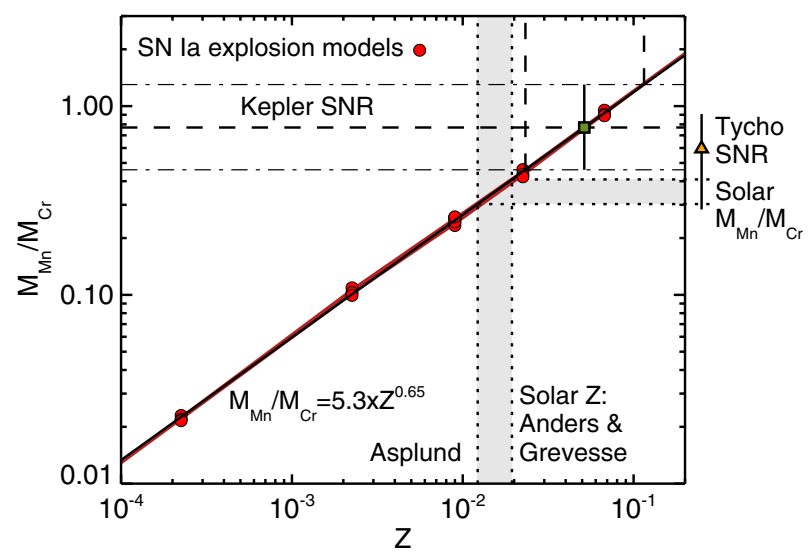

(b)

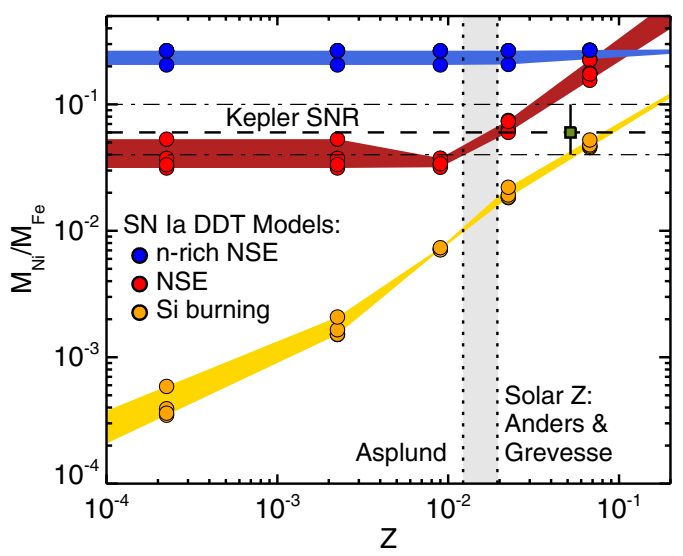

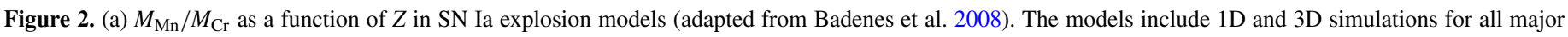

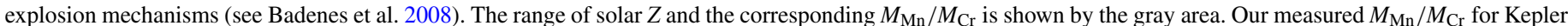

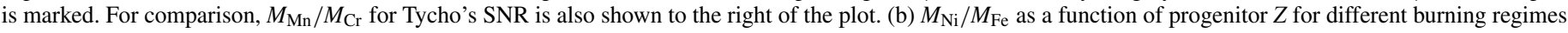
for delayed detonation SN Ia explosion models (the same models from Badenes et al. 2008). Our estimate for Kepler is shown.

\section{DISCUSSION AND CONCLUSIONS}

Our deep Suzaku XIS data allow us to accurately measure the $\mathrm{Fe} \mathrm{K} \alpha$ line center (Table 2), which constrains the ionization timescale to $n_{e} t \approx 2 \times 10^{10} \mathrm{~cm}^{-3} \mathrm{~s}$ for temperatures in the range $k T=3-8 \mathrm{keV}$ for the Fe-zone ejecta gas. The estimated Mn-to-Cr flux ratio is $f_{\mathrm{Mn}} / f_{\mathrm{Cr}}=0.60 \pm 0.16$ ( $2 \sigma$ uncertainties, hereafter). This flux ratio is $\sim 30 \%$ larger than that inferred for Tycho (Badenes et al. 2008; Tamagawa et al. 2009). The Mnto-Cr mass ratio can be calculated using their line flux ratio: $M_{\mathrm{Mn}} / M_{\mathrm{Cr}}=1.057\left(f_{\mathrm{Mn}} / f_{\mathrm{Cr}}\right) /\left(\varepsilon_{\mathrm{Mn}} / \varepsilon_{\mathrm{Cr}}\right)$, where $\varepsilon_{\mathrm{Mn}} / \varepsilon_{\mathrm{Cr}}$ is the ratio of specific emissivities per ion and 1.057 is the atomic mass ratio between $\mathrm{Mn}$ and $\mathrm{Cr}$ (Badenes et al. 2008). We calculate $\varepsilon_{\mathrm{Mn}} / \varepsilon_{\mathrm{Cr}}=0.82 \pm 0.20$ appropriate for the plasma with $k T=$ $3-8 \mathrm{keV}$ and $n_{e} t=2( \pm 0.03) \times 10^{10} \mathrm{~cm}^{-3} \mathrm{~s}$ using the new atomic data (K. A. Eriksen et al., in preparation) generated for this project with Flexible Atomic Code (Gu 2008). The uncertainty of $\varepsilon_{\mathrm{Mn}} / \varepsilon_{\mathrm{Cr}}$ depends on the measurements on the gas temperature, ionization timescale, and the atomic data. Based on our inferred ranges of $k T$ and $n_{e} t$ as well as the uncertainties of the atomic data, we estimate $\sim 20 \%-25 \%$ uncertainties on $\varepsilon_{\mathrm{Mn}} /$ $\varepsilon_{\mathrm{Cr}}$. Thus, we adopted a conservative limit of a $25 \%$ uncertainty on $\varepsilon_{\mathrm{Mn}} / \varepsilon_{\mathrm{Cr}}$. Then, we estimate $M_{\mathrm{Mn}} / M_{\mathrm{Cr}}=0.77_{-0.31}^{+0.53}$.

Assuming that the neutron excess in Kepler's progenitor was not significantly affected by the innermost NSE region, we may estimate the progenitor's $Z$ using a PL relation between $M_{\mathrm{Mn}} / M_{\mathrm{Cr}}$ and $Z$ (Badenes et al. 2008): i.e., unless the non-explosive C-burning, the so-called C-simmering (Piro \& Bildsten 2008), was widespread shortly before the SN explosion, $Z$ shows a simple relationship of $M_{\mathrm{Mn}} / M_{\mathrm{Cr}}=5.3 Z^{0.65}$ (Badenes et al. 2008). While C-simmering may modify the neutron excess in sub-luminous $\mathrm{SNe}$, where the explosive $\mathrm{Si}$ burning region extends deeper into the ejecta (Badenes et al. 2008), there is no evidence for a significantly sub-luminous explosion for Kepler in the historical light curve. Recent distance estimates of $d \gtrsim 6 \mathrm{kpc}$ (Aharonian et al. 2008; Patnaude et al. 2012) support a normal or luminous SN for Kepler. The Chandra X-ray spectrum of Kepler is consistent with an explosion model that produced $\sim 1 M_{\odot}$ of $\mathrm{Fe}$ (Patnaude et al. 2012). Thus, Kepler is unlikely the remnant of a sub-luminous SN. Then, we estimate that the progenitor's metallicity is $Z / Z_{\odot}=$ $3.6_{-2.0}^{+4.6}$ for $Z_{\odot}=0.014$ (Asplund et al. 2009) and 2.7 $7_{-1.5}^{+3.4}$ for $Z_{\odot}=0.019$ (Anders \& Grevesse 1989). This result provides the first observational evidence that the progenitor of an SN Ia was a star with a super-solar $Z$ (Figure 2(a)).

We detect the Ni K $\alpha$ line for the first time in Kepler. Since the bulk of $\mathrm{Fe}$ is created by the radioactive decay of ${ }^{56} \mathrm{Ni}$, $M_{\mathrm{Ni}} / M_{\mathrm{Fe}} \approx M^{58}{ }_{\mathrm{Ni}} / M^{56} \mathrm{Ni}=1.051\left(f_{\mathrm{Ni}} / f_{\mathrm{Fe}}\right) /\left(\varepsilon_{\mathrm{Ni}} / \varepsilon_{\mathrm{Fe}}\right)$, where 1.051 is the atomic mass ratio between ${ }^{58} \mathrm{Ni}$ and ${ }^{56} \mathrm{Fe}$. For the measured $f_{\mathrm{Ni}} / f_{\mathrm{Fe}}=0.031 \pm 0.005$ and $\varepsilon_{\mathrm{Ni}} / \varepsilon_{\mathrm{Fe}}=0.51 \pm 0.13$ for the $\mathrm{K} \alpha$ line in the same plasma condition discussed above, we estimate $M^{58} \mathrm{Ni} / M^{56} \mathrm{Ni} \sim 0.06_{-0.02}^{+0.04}$. This Ni mass ratio is in good agreement (within $\sim 10 \%$ ) with delayed detonation models, while being significantly different (by a factor of $>2$ ) from deflagration models (Iwamoto et al. 1999).

Our measured $M_{\mathrm{Ni}} / M_{\mathrm{Fe}}$ indicates that the bulk of the shocked Fe-group ejecta was synthesized in the explosive $\mathrm{Si}$ burning regime, especially considering the high $Z$ inferred from $M_{\mathrm{Mn}} / M_{\mathrm{Cr}}$ (Figure 2(b)). Contamination by a small amount of NSE material is possible, but a large contribution of products from the $n$-rich NSE regime deep in the core of the exploding star is ruled out, regardless of the $Z$ value. This strengthens our confidence on $M_{\mathrm{Mn}} / M_{\mathrm{Cr}}$ as a tracer of the neutron excess in the progenitor (and hence $Z$ ), since it is unlikely that material synthesized in the inner $n$-rich core can contaminate the shocked ejecta and increase $M_{\mathrm{Mn}} / M_{\mathrm{Cr}}$ while keeping $M_{\mathrm{Ni}} / M_{\mathrm{Fe}}$ at its observed value.

The observed $\mathrm{K} \alpha$ line flux ratio $f_{\mathrm{Ni}} / f_{\mathrm{Fe}}$ is higher (by $\sim 25 \%$ ) in the northern half of Kepler than in the southern half, while it is identical (within $\sim 2 \%$ ) between the eastern and western halves of the SNR. The Ni $\mathrm{K} \alpha$-to-Fe $\mathrm{K} \beta$ line flux ratio is also $\sim 70 \%$ higher in the northern half than in the south, while it is fully consistent between the east and west (within $\sim 2 \%$ ). The presence of the bright bow shock-like X-ray emission feature in the northern shell of Kepler suggests a more efficient development of the reverse shock there than in the southern shell. The reverse shock might have progressed further into the SNR in the northern shell (than in the south) to shock more Ni-rich ejecta close to the SNR center. Thus, although the spatial variation of the measured Ni-to-Fe line flux ratios is statistically marginal $(\sim 1.5 \sigma-2.5 \sigma)$, it would not be surprising if such a variation has a real physical origin. On the other hand, we do not find evidence for a similar spatial variation in the Mn-to-Cr line flux ratio. The Mn-to-Cr line flux ratio is consistent within $1 \sigma$ uncertainties between the north/south and the east/west halves of the SNR. This suggests that the correlation between 
the observed Mn-rich and Ni-rich ejecta is probably insignificant even if the Ni-rich ejecta were produced in the NSE. Thus, the bulk of the detected neutron excess appears to be associated with material synthesized in the explosive Si-burning regime of a relatively bright $\mathrm{SN}$ Ia, and it should be a tracer of the progenitor's $Z$.

The implied high $Z$ for the progenitor is consistent with the location of Kepler close to the Galactic center, where the interstellar chemical composition is expected to further enriched than in the solar neighborhood. Since higher- $Z$ stars likely result in a lower-mass core (Weiss \& Ferguson 2009), Kepler's progenitor WD probably went through an efficient mass growth before exploding as a relatively young prompt population SN Ia. A quantitative assessment of the suggested spatial variation of the Ni-to-Fe mass ratio and the effect from the core electron capture on the observed neutron excess may require detailed hydrodynamic calculations beyond the scope of this Letter.

The authors thank H. Kai and T. Yoshidome for their help with the XIS instrumental background investigation. This work has been supported in part by the NASA grants NNX09AV42G and NNX10AR47G. The work of K.M. has been partially supported by the Grant-in-Aid for Young Scientists (B) of the MEXT (No. 24740167). P.O.S. acknowledges partial support from NASA contract NAS8-03060.

\section{REFERENCES}

Aharonian, F., Akhperjanian, A. G., Barres de Almeida, U., et al. 2008, A\&A, 488,219

Anders, E., \& Grevesse, N. 1989, GeCoA, 53, 197

Asplund, M., Grevesse, N., Sauval, A. J., \& Scott, P. 2009, ARA\&A, 47, 481

Aubourg, É., Tojeiro, R., Jimenez, R., et al. 2008, A\&A, 492, 631
Baade, W. 1943, ApJ, 97, 119

Badenes, C., Borkowski, K. J., Hughes, J. P., Hwang, U., \& Bravo, E. 2006, ApJ, 645,1373

Badenes, C., Bravo, E., \& Hughes, J. P. 2008, ApJL, 680, L33

Bandiera, R. 1987, ApJ, 319, 885

Blair, W. P. 2005, in ASP Conf. Ser. 342, 1604-2004: Supernovae as Cosmological Lighthouses, ed. M. Turatto, S. Benetti, L. Zampieri, \& W. Shea (San Francisco, CA: ASP), 416

Blair, W. P., Long, K. S., \& Vancura, O. 1991, ApJ, 366, 484

Brachwitz, F., Dean, D. J., Hix, W. R., et al. 2000, ApJ, 536, 934

Bravo, E. 2013, A\&A, 550, A24

Burkey, M. T., Reynolds, S. P., Borkowski, K. J., \& Blondin, J. M. 2013, ApJ, 764, 63

Cassam-Chenai,, G., Decourchelle, A., Ballet, J., et al. 2004, A\&A, 414, 545

Chiotellis, A., Schure, K. M., \& Vink, J. 2012, A\&A, 537, 139

Decourchelle, A., \& Ballet, J. 1994, A\&A, 287, 206

Dennefeld, M. 1982, A\&A, 112, 215

Doggett, J. B., \& Branch, D. 1985, AJ, 90, 2303

Gu, M. F. 2008, CaJPh, 86, 675

Iwamoto, K., Brachwitz, F., Nomoto, K., et al. 1999, ApJS, 125, 439

Kinugasa, K., \& Tsunemi, H. 1999, PASJ, 51, 239

Krause, O., Tanaka, N., Usuda, T., et al. 2008, Natur, 456, 617

Kuchner, M. J., Kirshner, R. P., Pinto, P. A., \& Leibundgut, B. 1994, ApJL, 426, L89

Maoz, D., \& Mannucci, F. 2012, PASA, 29, 447

Park, S., Badenes, C., Hughes, J. P., et al. 2012, in AIP Conf. Proc. 1427, SUZAKU 2011: Exploring the X-Ray Universe: Suzaku and Beyond, ed. R. Petre, K. Mitsuda, \& L. Angelini (Melville, NY: AIP), 87

Patnaude, D. J., Badenes, C., Park, S., \& Laming, J. M. 2012, ApJ, 756, 6

Piro, A. L., \& Bildsten, L. 2008, ApJ, 673, 1009

Reynolds, S. P., Borkowski, K. J., Hwang, U., et al. 2007, ApJL, 668, L135

Reynoso, E. M., \& Goss, W. M. 1999, AJ, 118, 926

Sankrit, R., Blair, W. P., Delaney, T., et al. 2005, AdSpR, 35, 1027

Tamagawa, T., Hayato, A., Nakamura, S., et al. 2009, PASJ, 61, S167

Timmes, F. X., Brown, E. F., \& Truran, J. W. 2003, ApJL, 590, L83

Vink, J. 2008, ApJ, 689, 231

Weiss, A., \& Ferguson, J. W. 2009, A\&A, 508, 1343

Yang, X. J., Tsunemi, H., Lu, F. J., et al. 2013, ApJ, 766, 44 